\title{
Comparación del comportamiento glucémico de cuatro fórmulas nutricionales específicas para diabéticos mediante el uso de monitoreo continuo de glucosa
}

\author{
Comparison of the Glycemic Behavior of Four Specific Nutritional \\ Formulas for Diabetics with Continuous Glucose Monitoring
}

Wandurraga EA', Marín $L F^{2}$, Galeano $\mathrm{AM}^{3}$, Serrano-Gómez $S E^{4}$, Parra $G A^{5}$, Sarmiento JG .

${ }^{1}$ Endocrinólogo, Msc Oncología Molecular.

Profesor asociado Universidad Autónoma de Bucaramanga UNAB.

Endocrinólogos del Oriente (ENDORIENTE).

${ }^{2}$ Endocrinóloga

Centro Médico Carlos Ardila Lulle. Fundación Cardiovascular de

Colombia.

${ }^{3}$ Nutricionista dietista, Universidad Industrial de Santander

${ }^{4} \mathrm{MD}$, Magister en Epidemiología

Grupo Investigaciones Clínicas de Bucaramanga Universidad

Autónoma de Bucaramanga UNAB.

${ }^{5}$ Endocrinólogo

Profesor asociado Universidad Autónoma de Bucaramanga UNAB. Endocrinólogos del Oriente (ENDORIENTE).

${ }^{6}$ Endocrinólogo

Profesor asociado Universidad Autónoma de Bucaramanga UNAB. Endocrinólogos del Oriente (ENDORIENTE).

Autor de correspondencia

Edwin Antonio Wandurraga Sánchez

edwinwandurraga@gmail.com.

Fecha de recepción: 20/06/2018

Fecha de aceptación: 22/11/2018

\section{Resumen}

Introducción: Las fórmulas líquidas nutricionales son consideradas alimentos que dadas sus características aseguran un aporte de macro y micronutrientes estándar que pueden sustituir o complementar la alimentación de los pacientes con diabetes. No existen comparaciones frente a frente que evalúen el perfil glucémico entre fórmulas nutricionales específicas para diabéticos.

Materiales y métodos: Se realizó un ensayo clínico cruzado, con el fin de comparar el comportamiento glucémico y la variabilidad glucémica entre cuatro fórmulas nutricionales comerciales específicas para diabéticos y un desayuno están- dar en 10 sujetos con diabetes mellitus tipo 2, mediante el uso de un sistema de monitoreo continuo de glucosa. Se calculó el promedio, desviación estándar y el área bajo la curva en hiperglucemia de cada una de las fórmulas. Además se determinó el coeficiente de variación y otras medidas de variabilidad. Se analizó el delta de glucosa intersticial cada cinco minutos estratificado para cada fórmula nutricional.

Resultados: El promedio de glucosa para el desayuno control fue $146,7 \pm 44,3 \mathrm{mg} / \mathrm{dl}$, Ensoy Diabetes ${ }^{\circledR} 129,6 \pm 25,1 \mathrm{mg} /$ dl, Enterex DBT $^{\circledR} 129,6 \pm 26,8 \mathrm{mg} / \mathrm{dl}$, Glucerna $\mathrm{SR}^{\circledR} 131,5 \pm$ $31,7 \mathrm{mg} / \mathrm{dl}$ y Prowhey $\mathrm{DM}^{\circledR} 131,7 \pm 30,7 \mathrm{mg} / \mathrm{dl}$. Las cuatro fórmulas nutricionales tuvieron menor AUC en un periodo de cuatro horas (posabsortivo) respecto a un desayuno estándar $(p<0,001)$, generando menores excursiones glucémicas y reafirmando su menor índice glucémico.

Conclusiones: Las cuatro fórmulas nutricionales específicas para diabéticos evaluadas en nuestro estudio fueron superiores en comportamiento y variabilidad glucémica a un desayuno estándar en población diabética. Ensoy Diabetes ${ }^{\circledR}$ mostró menor AUC frente a Glucerna $\mathrm{SR}^{\circledR}$, hallazgo que podría ser explicado por el menor aporte real de carbohidratos de Ensoy Diabetes ${ }^{\circledR}$ (17 vs $22,4 \mathrm{~g}$ ).

Palabras clave: terapia nutricional, diabetes mellitus tipo 2, índice glucémico, monitoreo continuo de glucosa.

\section{Abstract}

Background: Liquid nutritional formulas ensure a contribution of standard macro and micronutrients that can substitute or complement the diet of patients with diabetes. There are no head-to-head comparisons that evaluate the glycemic profile between specific nutritional formulas for diabetics.

Methods: A cross-over clinical trial was conducted to compare the glycemic behavior and the glycemic variability be- 
tween four commercial specific nutritional formulas in diabetes and a standard breakfast in 10 subjects with type 2 diabetes mellitus, using a continuous glucose monitoring system. The average, standard deviation and the area under the curve (AUC) in hyperglycemia of each of the formulas were calculated. In addition, the coefficient of variation and other measures of variability were determined. The interstitial glucose delta was analyzed every 5 minutes stratified for each nutritional formula.

Results: Glucose average for standard breakfast was 146.7 $\pm 44.3 \mathrm{mg} / \mathrm{dl}$, Ensoy Diabetes $\AA 129.6 \pm 25.1 \mathrm{mg} / \mathrm{dl}$, Enterex DBT® $129.6 \pm 26.8 \mathrm{mg} / \mathrm{dl}$, Glucerna $S R \AA 131.5 \pm 31.7 \mathrm{mg} / \mathrm{dl}$ and Prowhey $D M ® 131.7 \pm 30.7 \mathrm{mg} / \mathrm{dl}$. The 4 nutritional formulas had lower AUC in a period of 4 hours (post-absorptive) compared to a standard breakfast ( $p<0.001)$, generating lower glycemic excursions and reaffirming their lower glycemic index.

Conclusions: When comparing formulas, they all had a good glycemic profile in general. Ensoy Diabetes ${ }^{\circledR}$ showed lower AUC compared to Glucerna $S R \circledast$, a finding that could be explained by the lower actual carbohydrate content of Ensoy Diabetes ${ }^{\circledR}(17$ vs $22.4 \mathrm{gr}$ ).

Key words: nutrition therapy, diabetes mellitus type 2, glycemic index, continuous glucose monitoring.

\section{Introducción}

La terapia nutricional es un pilar del tratamiento de la diabetes mellitus tipo 2 (DM2) ${ }^{(1,2)}$. Se trata de una estrategia que comprende la adopción de hábitos alimentarios saludables basados en un patrón bajo en carbohidratos, de alimentos con bajo índice glucémico y con un adecuado aporte de proteínas y grasas de acuerdo con el requerimiento energético diario, haciendo énfasis en el tamaño de las porciones. Esta aproximación busca contribuir al control metabólico, disminuir el riesgo de complicaciones metabólicas y alcanzar un peso saludable.

Las fórmulas líquidas o suplementos nutricionales son considerados alimentos que, dadas sus características, aseguran un aporte de macro y micronutrientes estándar y pueden sustituir o complementar la alimentación de los pacientes con diabetes. Estas fórmulas están elaboradas con una mezcla de carbohidratos complejos que aparentemente producen una respuesta más baja en las excursiones de glucosa posprandial. El estudio Look Ahead demostró que cuando se instauran cambios en el estilo de vida referentes a nutrición (incluyendo educación y suplementos nutricionales) se puede lograr reducción de HbA1c en un 0,62\% en población con DM2 con una respuesta sostenida a largo plazo $^{(3)}$.

Existe evidencia robusta que muestra el mejor comportamiento glucémico posprandial de las fórmulas específicas para diabetes frente a fórmulas nutricionales no específicas y frente a un desayuno estándar ${ }^{(4-7)}$. Sin embargo, no existen comparaciones frente a frente que evalúen el perfil glucémico entre fórmulas específicas para diabéticos.
El objetivo de este estudio fue realizar una comparación del comportamiento glucémico y de la variabilidad glucémica de cuatro fórmulas nutricionales comerciales específicas para diabéticos y un desayuno estándar en 10 sujetos con DM2, mediante el uso de un sistema de monitoreo continuo de glucosa (MCG).

\section{Materiales y métodos}

Se realizó un ensayo clínico cruzado para evaluar el comportamiento glucémico de cuatro fórmulas nutricionales para diabéticos disponibles en el mercado: Ensoy Diabetes ${ }^{\circledR}$, Enterex DBT ${ }^{\circledR}$ Glucerna $\mathrm{SR}^{\circledR}$ y Prowhey $\mathrm{DM}^{\circledR}$ en individuos con DM2 atendidos en un centro de endocrinología mediante el uso de monitoreo continuo de glucosa (MCG) de siete días (iPRO2- Medtronic ${ }^{\circledR}$ ). Los participantes recibían una fórmula nutricional diferente cada día, como sustituto del desayuno los cuatro primeros días y se comparaba con un desayuno estándar al quinto día. El contenido nutricional de las fórmulas se puede observar en el anexo 1.

Se incluyeron sujetos mayores de 30 años con diagnóstico de DM2 de al menos tres meses, en manejo solo con terapia nutricional y/o metformina y que tuvieran una HbA1c menor de $8,5 \%$. Por otro lado, mujeres embarazadas o en lactancia, pacientes con complicaciones microvasculares (retinopatía, nefropatía y neuropatía) y con consumo previo de algún suplemento nutricional en el último mes fueron excluidos.

Las fórmulas se prepararon el mismo día antes del consumo y de acuerdo con la recomendación establecida por el laboratorio fabricante. Cada paciente debió asistir de lunes a viernes al centro para: día 1 colocación del MCG y entrenamiento sobre el protocolo, días 2 a 5 para la administración de una fórmula nutricional y sesión de educación. Debía permanecer en reposo cuatro horas luego de la ingesta. Días 6 y 7 permanecía en casa para tomar un desayuno usual (control), y el día 8 asistir nuevamente al centro para retiro del MCG y descarga de datos. El protocolo realizado se encuentra detallado en el anexo 2 .

Las medidas de variabilidad glucémica calculadas y analizadas a partir de la descarga de datos del MCG fueron: promedio de glucosa con desviación estándar (DE), coeficiente de variación (CV), rango intercuartílico (IQR), amplitud media de la excursión glucémica (MAGE) y área bajo la curva (AUC) en hiperglicemia mayor a 140,150,160 y $180 \mathrm{mg} / \mathrm{dl}$. Para determinar el comportamiento glucémico posterior al consumo de la fórmula nutricional y del desayuno control se evaluaron las cuatro horas posteriores al momento de la administración de la fórmula. Adicionalmente, se interrogó por posibles efectos adversos relacionados con la ingesta y preferencia de sabor de cada fórmula. El paciente desconocía el nombre de cada producto.

El estudio fue aprobado por el comité de ética de la institución y se aplicó consentimiento informado a todos los participantes. 


\section{Análisis estadístico}

Se realizó un análisis univariado de las medidas descriptivas sociodemográficas y un análisis bivariado, calculando promedio, desviación estándar, CV, IQR, MAGE y el área bajo la curva en hiperglicemia de cada una de las fórmulas. Se realizó una prueba de ANOVA con un postest de Bonferroni, comparando el promedio de glucosa de las cuatro fórmulas para evaluar diferencias entre ellas.

Se analizó el delta de glucosa intersticial cada cinco minutos, estratificado para cada fórmula nutricional. Todos los datos se analizaron en Stata 14.

\section{Resultados}

Diez pacientes fueron incluidos en el estudio, siete mujeres y tres hombres con un promedio de edad de $63,3 \pm 12$ años y 3,8 $\pm 2,9$ años de duración de la diabetes. La HbA1c promedio fue de $6,7 \pm 0,9 \%$. Ocho pacientes estaban en terapia con metformina. Todos los pacientes recibieron y toleraron las cuatro fórmulas nutricionales y realizaron el desayuno estándar. La calibración del MCG pudo hacerse de forma exitosa, teniendo en cuenta los datos de glucometría en el $100 \%$ de los momentos exigidos por el protocolo. No hubo pérdida de datos del sensor del MCG.

La tabla 1 muestra las medidas de variabilidad glucémica evaluadas durante un periodo de cuatro horas posterior a la ingesta de la fórmula nutricional y del desayuno estándar. El comportamiento glucémico evaluado como AUC en hiperglucemia mayor a $140 \mathrm{mg} / \mathrm{dl}, 160 \mathrm{mg} / \mathrm{dl}$ y $180 \mathrm{mg} / \mathrm{dl}$ se muestra en la tabla 2.

La figura 1 muestra el comportamiento glucémico desde el momento de la toma de la fórmula nutricional (momento 0) y las cuatro horas posteriores, mostrando el delta de glucosa en relación con el tiempo. Se observó diferencia estadísticamente significativa entre el AUC del desayuno control con las todas las fórmulas.

Las cuatro fórmulas fueron evaluadas para detectar posibles efectos secundarios luego de la ingesta. Tres pacientes refirieron algún síntoma luego del consumo de Enterex DBT ${ }^{\circledR}$, incluyendo náusea, cefalea, distensión abdominal, mareo o diarrea. Dos pacientes reportaron cefalea luego del consumo de Prowhey $\mathrm{DM}^{\circledR}$ y dos pacientes refirieron diarrea y dolor o distensión abdominal tras la administración de Ensoy Diabe-

Tabla 1. Medidas de variabilidad glucémica durante cuatro horas luego de administrar las fórmulas nutricionales y el desayuno estándar

\begin{tabular}{|c|c|c|c|c|c|c|}
\hline Fórmula & $\begin{array}{c}\text { Promedio } \\
\text { (mg/dl) }\end{array}$ & $\begin{array}{c}\text { DE } \\
(\mathrm{mg} / \mathrm{dl})\end{array}$ & CV & $\begin{array}{c}\text { p25-P75 } \\
\text { (mg/dl) }\end{array}$ & $\begin{array}{c}\text { IQR } \\
\text { (mg/dl) }\end{array}$ & $\begin{array}{c}\text { MAGE } \\
\text { (mg/dl) }\end{array}$ \\
\hline $\begin{array}{l}\text { Control } \\
\text { (desayuno } \\
\text { estándar) }\end{array}$ & $146,7^{*}$ & $44,3^{*}$ & $0,30^{*}$ & $117-167^{*}$ & $50^{*}$ & $53,1^{*}$ \\
\hline Ensoy Diabetes ${ }^{\circledast}$ & 129,6 & 25,1 & 0,19 & $112-145$ & 33 & 39,4 \\
\hline Enterex DBT ${ }^{\circledR}$ & 129,6 & 26,8 & 0,20 & $111-144$ & 33 & 40,8 \\
\hline Glucerna SR ${ }^{\circledast}$ & 131,5 & 31,7 & 0,24 & $106-148$ & 42 & 41,2 \\
\hline Prowhey DM ${ }^{\circledR}$ & 131,7 & 30,7 & 0,23 & $108-149$ & 41 & 40,6 \\
\hline
\end{tabular}

* Valor de $p<0,001$ comparado con las cuatro fórmulas nutricionales.

DE: desviación estándar. CV: coeficiente de variación. IQR: rango intercuartílico. MAGE: amplitud media de la excursión glucémica.

Tabla 2. Comportamiento de AUC luego de administrar las fórmulas nutricionales y el desayuno estándar

\begin{tabular}{|c|c|c|c|}
\hline Fórmula nutricional & $\begin{array}{c}\text { AUC } \\
>140 \mathrm{mg} / \mathrm{dl}\end{array}$ & $\begin{array}{c}\text { AUC } \\
>160 \mathrm{mg} / \mathrm{dl}\end{array}$ & $\begin{array}{c}\text { AUC } \\
>180 \mathrm{mg} / \mathrm{dl}\end{array}$ \\
\hline Control & $41,5^{*}$ & $41,7^{\dagger}$ & $48,6^{*}$ \\
\hline Ensoy Diabetes ${ }^{\circledast}$ & $19,8^{\ddagger}$ & $20,5^{\S}$ & 11,3 \\
\hline Enterex DBT ${ }^{\circledR}$ & 23,7 & 21,5 & 19,0 \\
\hline Glucerna SR $^{\circledR}$ & 30,0 & 33,8 & 24,1 \\
\hline Prowhey DM ${ }^{\circledast}$ & 27,8 & 24,1 & 21,8 \\
\hline
\end{tabular}

* $\mathrm{p}<0,001$ respecto a las demás fórmulas nutricionales. ${ }^{\dagger} \mathrm{p}<0,001$ respecto a fórmula nutricional Ensoy Diabetes, Enterex DBT y Prowhey DM. ${ }^{\ddagger} \mathrm{p}=0,01$ respecto a fórmula nutricional Glucerna SR. ${ }^{\S} \mathrm{p}<0,05$ respecto a fórmula nutricional Glucerna SR. AUC: área bajo la curva. 
Figura 1. Delta de glucosa luego de ingesta de cuatro fórmulas nutricionales y desayuno estándar

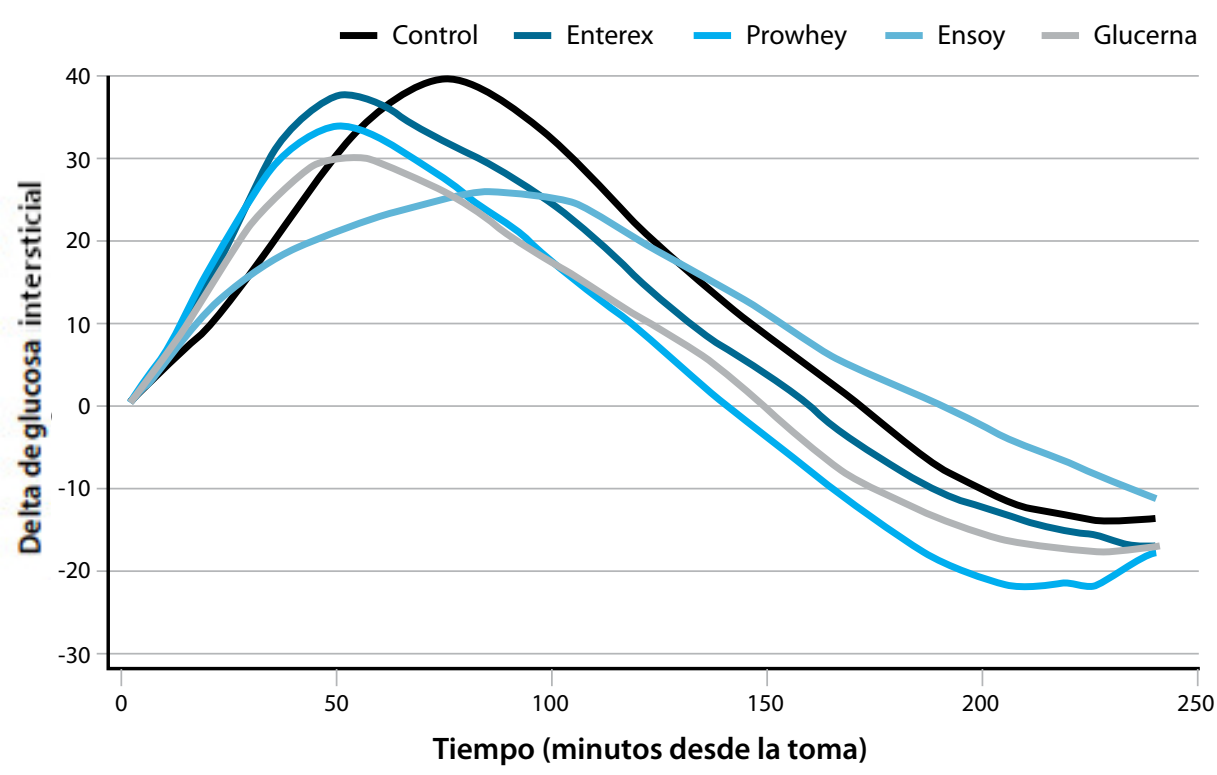

* Valor de $p<0,001$ comparando el control con las cuatro fórmulas nutricionales.

tes ${ }^{\circledR}$. Para Glucerna SR ${ }^{\circledR}$ no se reportó ningún síntoma asociado.

Respecto a la preferencia del sabor para las fórmulas, Glucerna $\mathrm{SR}^{\circledR}$ recibió 8 votos, Enterex DBT ${ }^{\circledR} 4$ votos, Prowhey $\mathrm{DM}^{\circledR} 1$ voto y no hubo preferencia por Ensoy Diabetes ${ }^{\circledR}$. Dos pacientes reportaron más de una preferencia. Cuando se interrogó por cuál fórmula compraría, cuatro pacientes eligieron Glucerna $\mathrm{SR}^{\circledR}$, cuatro pacientes Enterex DBT ${ }^{\circledR}$ y un paciente Ensoy Diabetes ${ }^{\circledR}$. Un paciente no respondió.

\section{Discusión}

Las diferentes fórmulas específicas para diabetes evaluadas en nuestro estudio tuvieron menor AUC en un periodo de cuatro horas (posabsortivo) respecto a un desayuno estándar con diferencia estadísticamente significativa, generando menores excursiones glucémicas y reafirmando su menor índice glucémico. Lo anterior puede ser explicado por una menor cantidad y tipo de carbohidrato empleado en la fórmula respecto al contenido en una comida convencional. Mientras que una "dosis" de suplemento nutricional contiene entre 25 y $33 \mathrm{~g}$ de carbohidrato total, un desayuno usual en nuestro país puede alcanzar fácilmente 45 a 75 gramos. Además, carbohidratos modificados como la maltodextrina resistente, utilizados por algunas fórmulas nutricionales puede favorecer un mejor comportamiento glucémico ${ }^{(8)}$. De esta manera, las fórmulas nutricionales específicas para diabetes pueden ser utilizadas como sustitutos a una comida, principalmente en aquel paciente con DM2 e hiperglicemia posprandial y/o exceso de peso.

Respecto al comportamiento glucémico entre las cuatro fórmulas medido por AUC mayor a 140, 160 y 180 mg/dl encon- tramos un mejor perfil glucémico de todas las fórmulas con respecto al desayuno estándar y de Ensoy Diabetes frente a Glucerna $\mathrm{SR}^{\circledR}$ en AUC mayor a 140 y $160 \mathrm{mg} / \mathrm{dl}$, lo que puede ser explicado por el menor aporte real de carbohidratos de Ensoy Diabetes ${ }^{\circledR}$ (17 vs. 22,4 g). De igual forma, al evaluar el delta de glucosa desde el momento de la administración de la fórmula o del desayuno usual, el AUC fue menor para las cuatro fórmulas. El desayuno control mostró la mayor excursión glucémica posprandial. De forma llamativa, luego del pico de glucemia posprandial, las cuatro fórmulas y el desayuno control mostraron descenso de la glucosa hasta valores inferiores al momento basal (hasta $-20 \mathrm{mg} / \mathrm{dl}$ ), esto puede ser explicado por la respuesta insulínica a la glucosa contenida en el carbohidrato del alimento y a una respuesta incretínica que aún persiste en los pacientes con DM2 temprana. Este hallazgo fue similar al demostrado por Gulati y $\operatorname{cols}^{(6)}$, donde los pacientes tenían un delta de glucosa de $-13 \mathrm{mg} / \mathrm{dl}$ a las tres horas posprandial respecto a la glucemia basal luego de la ingesta de un desayuno convencional o una dieta específica para DM2 (Nutren Diabetes ${ }^{\circledR}$ ).

$\mathrm{Al}$ comparar la variabilidad glucémica medida por $\mathrm{DE}, \mathrm{CV}$, IQR o MAGE no se encontraron diferencias entre las cuatro fórmulas. Teniendo en cuenta un valor normal de CV entre 0,19 y 0,25 y de MAGE entre 41 y $48 \mathrm{mg} / \mathrm{dl}^{(9)}$, la respuesta glucémica a la ingesta de todas las fórmulas fue poco variable. Sin embargo, considerando una DE normal entre 10 y $26 \mathrm{mg} / \mathrm{dl}$, Enterex $\mathrm{DBT}^{\circledR}$, Glucerna $\mathrm{SR}^{\circledR}$ y Prowhey $\mathrm{DM}^{\circledR}$ mostraron variabilidad glucémica aumentada.

Nuestro estudio es el primer ensayo clínico que compara directamente cuatro fórmulas nutricionales para esta población. Corroboramos la información de seguridad glucémica y aportamos evidencia al clínico y a los pacientes para el uso seguro de esta terapia. Teniendo en cuenta el comportamiento glucémico similar de las cuatro fórmulas, la preferencia entre ellas puede ser basada en el sabor, la tolerancia gastrointestinal, los efectos adversos, el contenido de otros nutrientes y el costo.

Una debilidad de nuestro estudio fue la muestra de 10 pacientes, sin embargo, teniendo en cuenta que el uso de MCG aporta medición de glucosa intersticial cada cinco minutos, el número de observaciones es muy significativo para evaluar el periodo posprandial. Además, cada paciente fue su propio control al recibir las cuatro fórmulas y el desayuno estándar según lo estipulado en el protocolo. 


\section{Conclusiones}

Las cuatro fórmulas nutricionales específicas para diabéticos evaluadas en nuestro estudio fueron superiores en comportamiento y variabilidad glucémica a un desayuno estándar en población diabética previamente educada en medidas nutricionales. Al comparar entre fórmulas, todas tuvieron en general un buen perfil glucémico. Ensoy Diabetes ${ }^{\circledR}$ mostró menor AUC frente a Glucerna $\mathrm{SR}^{\circledR}$, hallazgo que podría ser explicado por el menor aporte real de carbohidratos de Ensoy Diabetes ${ }^{\circledR}$.

\section{Conflictos de interés y financiación}

Boydorr Nutrition proporcionó las 10 fórmulas nutricionales de Prowhey DM ${ }^{\circledR}$ y Victus las 10 fórmulas nutricionales de Enterex DBT ${ }^{\circledR}$. Sin embargo, ninguna casa farmacéutica estuvo involucrada en el desarrollo del protocolo ni en el análisis de datos o en la realización del manuscrito. EAWS fue conferencista de Abbott Nutrition y AMGR es conferencista de Abbott Nutrition. Los demás autores se declaran libres de conflictos de interés. Para la ejecución del monitoreo continuo de glucosa hubo financiación por parte de la Asociación Colombiana de Endocrinología, Diabetes \& Metabolismo.

\section{Agradecimientos}

A los pacientes participantes y a la Asociación Colombiana de Endocrinología (ACE) por la financiación para el estudio. A Boydorr Nutrition y a Victus por suministrar los 10 suplemen-

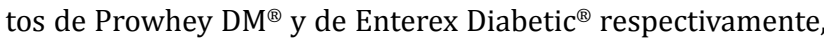
para el estudio.

ANEXO 1. Información nutricional de las cuatro fórmulas específicas para diabetes (Porción sugerida por la casa fabricante)

\begin{tabular}{l|c|c|c|c} 
& Ensoy Diabetes & & Prowhey DM $^{\odot}$ \\
\hline Kcal & 210 & Enterex DBT & Glucerna SR \\
\hline Proteína (g) & 12 & 240 & 222 & 15 \\
\hline Grasa (g) & 10 & 12 & 8 & 10 \\
\hline Carbohidrato total (g) & 30 & 9 & 27,5 & 22 \\
\hline & Maltitol & 27 & Maltodextrina \\
Tipo de carbohidrato & Polidextrosa & Maltodextrina & Sucromalt & Sucralosa \\
\hline Alcoholes (g) & 26 & - & 5,5 & - \\
\hline Fibra (g) & 4 & 3 & 5 & 3 \\
\hline Aporte real de \\
carbohidrato (gr) & 17 & 27 & 22,4 & 22 \\
\hline Vitamina E (mg) & 3.3 & 12 & 4,3 & 10 \\
\hline Vitamina C (mg) & 54 & 60 & 21,3 & 354 \\
\hline Ácido fólico (mcg) & 120 & 100 & 59 & 420 \\
\hline Cromo (mcg) & 18 & 60 & 200 & 48 \\
\hline
\end{tabular}

ANEXO 2. Protocolo de comparación de cuatro fórmulas nutricionales

Día 1 (lunes): Se citó al paciente en horas de la tarde para la instalación del sistema de MCG y explicación de los cuidados que debe tener en cuenta. El paciente debía tomar tres glucometrías: 1. A la hora de instalado el MCG 2. A las tres horas de instalado el MCG o a las dos horas después de la primera glucometría; y 3. A las 12 horas. El MCG se dejó instalado durante siete días.

Día 2 (martes): Se realizó la primera toma de la fórmula nutricional y una vez consumida permaneció sentado durante las cuatro horas siguientes sin realizar alguna actividad que requiriera esfuerzo físico. En este tiempo se realizó una encuesta sociodemográfica, recordatorio de 24 horas y frecuencia de consumo. Así mismo durante estas cuatro horas se realizó toma de glucometrías a los 0 min (antes de consumir la fórmula) a los 30 min (después de consumir la fórmula), 60 min, 2 horas, 3 horas y 4 horas. Adicionalmente el paciente realizó glucometrías antes de la comida y antes de dormir.

Día 3 (miércoles): Se realizó la segunda toma de la fórmula nutricional y se estableció la misma metodología del día dos; el tiempo en que debían permanecer sin esfuerzo físico se destinó a educación en diabetes. 
Día 4 (jueves): Se realizó la ingesta de la tercera fórmula nutricional, siguiendo el mismo procedimiento del día 2; el tiempo en que debían permanecer sin esfuerzo físico se destinó a educación en diabetes.

Día 5 (viernes): Se realizó la ingesta de la cuarta fórmula nutricional y se estableció la misma metodología del día 2; el tiempo en que debían permanecer sin esfuerzo físico se destinó a educación en diabetes.

Día 6 (sábado): El paciente no asistió al centro y desayunó en casa como normalmente, tomando glucometría antes de cada comida principal (desayuno, almuerzo y comida). Se recomendó reposo durante cuatro horas después de la ingesta del desayuno.

Día 7 (domingo): El paciente no asistió al centro y desayunó en casa como normalmente, tomando glucometría antes de cada comida principal (desayuno, almuerzo y comida). Se recomendó reposo durante cuatro horas después de la ingesta.

Día 8 (lunes de la siguiente semana): El paciente asistió al centro en horas de la mañana para retiro del MCG y posterior descarga de datos.

Nota: Todas las glucometrías se realizaron con un glucómetro Fastcheck.

\section{Preparación de fórmulas nutricionales}

Las fórmulas nutricionales que se escogieron para evaluar su comportamiento glucémico fueron representativas del mercado. Las fórmulas se prepararon el mismo día antes del consumo y de acuerdo con la recomendación establecida por el laboratorio fabricante.

- GLUCERNA SR: disolver 6 medidas de la cuchara medidora que trae la lata en $200 \mathrm{ml}$ de agua

- ENSOY DIABETES: disolver 4 medidas de la cuchara medidora que trae la lata en $190 \mathrm{ml}$ de agua

- PROWHEY DM: disolver 4 medidas de la cuchara medidora que trae la lata en $200 \mathrm{ml}$ de agua

- ENTEREX DBT: Viene lista para consumo

\section{Consumo de fórmulas nutricionales}

Los participantes del proyecto consumieron las fórmulas una por día en los días 2, 3, 4 y 5. Asistieron en horas de la mañana con un ayuno nocturno mínimo de 6 horas y previo al consumo la glucometría al minuto cero debía ser menor a $180 \mathrm{mg} / \mathrm{dl}$. Consumieron la totalidad de la fórmula nutricional sin alimentos adicionales en las siguientes cuatro horas.

\section{Educación en diabetes}

La educación al paciente con diabetes se llevó a cabo durante el periodo de reposo luego del consumo de la fórmula nutricional. Se abordaron los siguientes puntos en tres sesiones a manera de talleres.

- Generalidades de la diabetes: fisiopatología, síntomas, diagnóstico, complicaciones, control y cuidados en general.

- Alimentación en diabetes: grupos de alimentos, distribución, tiempos de comida, horarios, porciones.

\section{Referencias}

1. American Diabetes Association. Lifestyle Management: Standards of Medical Care in Diabetes-2018. Diabetes Care. 2018 Jan;41(Suppl 1):S38-S50.

2. Aschner P, Muñoz OM, Girón DM, García OM, Fernández DG, Casas LA, et. al. Guía de práctica clínica para el diagnóstico, tratamiento y seguimiento de la diabetes mellitus tipo 2 en la población mayor de 18 años. Guía No. GPC-2015-51. Colombia Médica 2016; 47 (2):109-31.

3. Look AHEAD Research Group, Wing RR, Bolin P, Brancati FL, Bray GA, Clark JM. Cardiovascular effects of intensive lifestyle intervention in type 2 diabetes. N Engl J Med. 2013 Jul 11;369(2):145-54.

4. Elia M, Ceriello A, Laube H, Sinclair AJ, Engfer M, Stratton RJ. Enteral nutritional support and use of diabetes-specific formulas for patients with diabetes: a systematic review and meta-analysis. Diabetes Care. 2005 Sep;28(9):2267-79.

5. Ojo 0, Brooke J. Evaluation of the role of enteral nutrition in managing patients with diabetes: a systematic review. Nutrients. 2014 Nov 18;6(11):5142-52.

6. Gulati S, Misra A, Nanda K, Pandey RM, Garg V, Ganguly S, Cheung L. Efficacy and tolerance of a diabetes specific formula in patients with type 2 diabetes mellitus: An open label, randomized, crossover study. Diabetes Metab Syndr. 2015 Oct-Dec;9(4):252-7

7. Stenvers DJ, Schouten LJ, Jurgens J, Endert E, Kalsbeek A, Fliers E, Bisschop PH. Breakfast replacement with a low-glycaemic response liquid formula in patients with type 2 diabetes: a randomised clinical trial. Br J Nutr. 2014 Aug 28;112(4):504-12.

8. Livesey G1, Tagami H. Interventions to lower the glycemic response to carbohydrate foods with a low-viscosity fiber (resistant maltodextrin): meta-analysis of randomized controlled trials. Am J Clin Nutr. 2009 Jan;89(1):114-25.

9. Kovatchev B, Cobelli C. Glucose Variability: Timing, Risk Analysis, and Relationship to Hypoglycemia in Diabetes. Diabetes Care. 2016 Apr;39(4):502-10. 